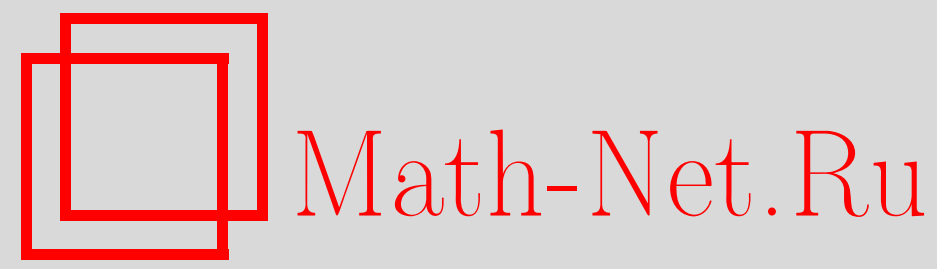

С. А. Егишянц, Е. И. Островский, Локальные и глобальные верхние функции для случайных полей, Теория вероятн. и ее примен., 1996, том 41, выпуск 4, 755-764

DOI: https://doi.org/10.4213/tvp3200

Использование Общероссийского математического портала Math-Net.Ru подразумевает, что вы прочитали и согласны с пользовательским соглашением http://www.mathnet.ru/rus/agreement

Параметры загрузки:

IP : 54.157 .27 .8

26 апреля 2023 г., 12:10:54

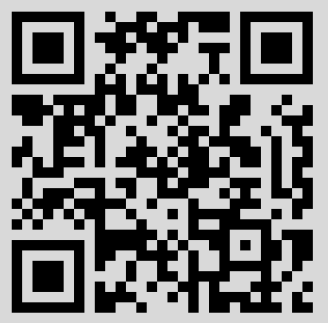




\title{
ЛОКАЛЬНЫЕ И ГЛОБАЛЬНЫЕ ВЕРХНИЕ ФУНКЦИИ ДЛЯ СЛУЧАЙНЫХ ПОЛЕЙ
}

\begin{abstract}
Для произвольных, т.е. необязательно гауссовских случайных полей, вводятся и вычисляются локальные и глобальные верхние функции. На рассматриваемое поле налагается только условие Крамера. Несмотря на такую общность, на примерах показано, что результаты точны и для гауссовских полей, изученных ранее. Oписаны также возможные приложения.
\end{abstract}

Ключевые слова и фразы: случайное поле, локальные и глобальные модули непрерывности, преобразование Юнга-Фенхеля, метрическая энтропия, экспоненциальная оценка.

1. Введение. Пусть $\xi(t)$ - сепарабельное центрированное случайное поле, определенное на некотором, помимо вероятностного, метрическом пространстве $(T, d), t \in T$. Глобальные и локальные модули непрерывности $\xi(t)$ вводятся, как обычно, формулами

$$
\begin{array}{ll}
\Delta(h)=\sup \{|\xi(t)-\xi(s)|, t, s: d(t, s) \leqslant h\}, & h>0, \\
\omega(h)=\sup \left\{\left|\xi(t)-\xi\left(t_{0}\right)\right|, t: d\left(t, t_{0}\right) \leqslant h\right\}, & t_{0} \in T .
\end{array}
$$

Неслучайная функщия $f(h), f(h)>0$, называется глобальной верхней функцией для $\xi(t)$, если для некоторой константы $C_{1}>0$ с вероятностью 1 выполнено неравенство $\lim \sup _{h \rightarrow 0+}(\Delta(h) / f(h)) \leqslant C_{1}$. Аналогично, неслучайная функция $g(h), g(h)>0$, называется локальной верхней функцией, если $\lim \sup _{h \rightarrow 0+}(\omega(h) / g(h)) \leqslant C_{2}$.

Задача отыскания верхних функций для случайных процессов и полей является классической. Достаточно вспомнить законы повторного логарифма для винеровского процесса или поля [1, с. 224], для сумм независимых случайных величин [2, с. 358], для гауссовских процессов и полей [3] и др. Из последних результатов, относяшихся к гауссовскому случаю, отметим работу Альбина [4], из результатов для негауссовских. полей - работы Дмитровского [5], Ферника [6].

Цель статьи - вычисление функций $f(h)$ и $g(h)$ для полей, удовлетворяющих только условию Крамера. Полученные результаты для

*Обнинский институт атомной энергии, Студгородок, 1, 249020 Обнинск, Россия. 
гауссовских полей $\xi(t)$ совпадают с известными, для негауссовских уточняют [5] и [6]; в частности, в [5] функция $f(h)$ не совпадает с наилучшей для винеровского процесса, в [6] $f(h)$ не точна для гауссовского стационарного процесса.

Параллельно получаются полезные в приложениях экспоненциальные оценки вероятностей вида

$$
P_{G}(u)=\mathbf{P}\left\{\sup _{h>0} \frac{\Delta(h)}{f(h)}>u\right\}, \quad P_{L}(u)=\mathbf{P}\left\{\sup _{h>0} \frac{\omega(h)}{g(h)}>u\right\} .
$$

Введем необходимые обозначения. Положим

$$
\varphi(\lambda)=\sup _{t \in T} \log \mathrm{E} \exp \lambda \xi(t) .
$$

Предполагается, что $\varphi(\lambda)<\infty, \lambda \in \mathbf{R}^{1}$, и что $\lim \varphi(\lambda) / \lambda=\infty,|\lambda| \rightarrow \infty$. C помошью $\varphi(\lambda)$ вводится, согласно [7], банахово пространство случайных величин $B(\varphi)$. Напомним для удобства читателя, что норма в $B(\varphi)$ опредепяется формулой

$$
\|\xi\|=\sup _{\lambda \neq 0} \frac{\varphi^{-1}(\log \mathbf{E} \exp \lambda \xi)}{|\lambda|}
$$

так что $\mathbf{E} \exp \lambda \xi \leqslant \exp \varphi(\lambda\|\xi\|), \forall \lambda \in \mathbf{R}^{1}$. Отметим, что в дальнейшем можно вместо функции $\varphi(\lambda)$ брать любую ее четную выпуклую мажоранту, монотонно возрастаюшую при $\lambda>0$, равную нулю при $\lambda=0$. Преобразование Юнга-Фенхеля функции $\varphi(\lambda)$ обозначим через $\psi(x)$ :

$$
\psi(x)=\sup _{\lambda \in \mathbf{R}^{1}}(\lambda x-\varphi(\lambda)) .
$$

Определим также $\chi(x)=\varphi^{-1}(x)$ - обратную функцию к $\varphi(x)$ при $x \geqslant 0$. Естественную метрику $d(t, s)$ на множестве $T$ определим выражением $d(t, s)=\|\xi(t)-\xi(s)\|$. Наименьшее число $d$-шаров радиуса $\varepsilon>0$, покрывающих некоторое подмножество $S$ пространства $T, S \subset T$, обозначим $N(S, d, \varepsilon)=N(S, \varepsilon)$. Энтропией $S$ называется логарифм $N$ :

$$
\mathcal{H}(S, \varepsilon)=\log N(S, \varepsilon) .
$$

Пусть $B\left(s_{0}, \delta\right)=\left\{t: t \in T, d\left(t, s_{0}\right) \leqslant \delta\right\}-d$-шар радиуса $\delta$ с центром в точке $s_{0}$. Поле $\xi(t)$ называется (локально) однородным в фиксированной точке $t_{0}, t_{0} \in T$, если для любого $\varepsilon>0$

$$
\mathcal{H}_{1}(\varepsilon)=\sup _{\delta>0} \mathcal{H}\left(B\left(t_{0}, \delta\right), \delta \varepsilon\right)<\infty
$$

Поле $\xi(t)$ называется (локально) однородным на всем множестве $T$, если для любого $\varepsilon>0$

$$
\mathcal{H}_{2}(\varepsilon)=\sup _{t_{0} \in T} \sup _{\delta>0} \mathcal{H}\left(B\left(t_{0}, \delta\right), \delta \varepsilon\right)<\infty
$$


Если $T$ - ограниченное выпуклое подмножество $\mathbf{R}^{k}$ и $d(t, s) \sim|t-s|^{\alpha}$, $t-s \rightarrow 0, \alpha>0$, то оба условия (1) и (2) выполнены.

2. Локальные верхние функции. Положим

$$
M_{1}=\int_{0}^{1} \frac{\mathcal{H}_{1}(x)}{\chi\left(H_{1}(x)\right)} d x
$$

Сходимость интеграла (3) есть достаточное условие непрерывности $\xi(t)$ в некоторой $d$-окрестности точки $t_{0}[7]$.

Теорема 1. Пусть поле $\xi(t)$ локально однородно в точке $t_{0}$, $t_{0} \in T$, и сходится интеграл (3). Положим $g(h)=h \psi^{-1}(\log (\log (1 / h)+$ е)). Тогда с вероятностью 1

$$
\limsup _{h \rightarrow 0+} \frac{\omega(h)}{g(h)} \leqslant 1 .
$$

При этом

$$
P_{L}(u)=\mathbf{P}\left\{\sup _{h \in(0,1)} \frac{\omega(h)}{g(h)}>u\right\} \leqslant C_{5} e^{-\psi\left(C_{4} u\right)}
$$

Здесь и далее $C_{i}-$ константы, не зависящие от $u, u \geqslant u_{0}>0$.

Д о к а 3 а т е л ь с т в о сходно с выводом закона повторного логарифма для винеровского процесса. Пусть $a-$ произвольное число из интервала $(0,1)$. Положим

$$
\begin{aligned}
T(n) & =\left\{t: t \in T, a^{n+1} \leqslant d\left(t, t_{0}\right)<a^{n}\right\}, \quad n=0,1,2, \ldots, \\
B(n) & =B\left(t_{0}, a^{n}\right), \quad \eta_{n}(t)=a^{-n}\left(\xi(t)-\xi\left(t_{0}\right)\right), \\
P_{n}(u) & =\mathbf{P}\left\{\sup _{t \in T(n)}\left|\eta_{n}(t)\right|>u a \psi^{-1}\left(\log \left(\log a^{-n}+e\right)\right)\right\} \\
d_{n}(t, s) & =\left\|\eta_{n}(t)-\eta_{n}(s)\right\|, \quad t, s \in T(n), \\
K(n) & =\int_{0}^{1} \frac{\mathcal{H}\left(T(n), d_{n}, x\right)}{\chi\left(\mathcal{H}\left(T(n), d_{n}, x\right)\right)} d x .
\end{aligned}
$$

В силу локальной однородности $\xi(t)$ в точке $t_{0}$

$$
\begin{aligned}
\mathcal{H}\left(T(n), d_{n}, \varepsilon\right) & =\mathcal{H}\left(T(n), d a^{-n}, \varepsilon\right) \leqslant \mathcal{H}\left(B(n), d a^{-n}, \varepsilon\right) \\
& =\mathcal{H}\left(B(n), d, \varepsilon a^{n}\right)=\mathcal{H}\left(B\left(t_{0}, a^{n}\right), d, \varepsilon a^{n}\right) \leqslant \mathcal{H}_{1}(\varepsilon) .
\end{aligned}
$$

Поэтому

$$
K(n) \leqslant \int_{0}^{1} \frac{H_{1}(x)}{\chi\left(H_{1}(x)\right)} d x=M_{1}<\infty .
$$

Так как $\left\|\eta_{n}(t)\right\| \leqslant 1, t \in T(n)$, то для оценки вероятности $P_{n}(u)$ можно применить неравенство Дмитровского (см. [5], [7]): шри условии, что 
$u a \psi^{-1}\left(\log \left(\log a^{-n}+e\right)\right) \geqslant C_{6}, C_{6}=C_{6}(\varphi)$, имеем:

$$
P_{n}(u) \leqslant 2 \exp \left\{-\psi\left(v\left(1-\frac{2 \sqrt{2 M_{1}}}{\sqrt{v}}\right)\right)\right\},
$$

где $v=a u \psi^{-1}\left(\log \left(\log a^{-n}+e\right)\right)$, или (обозначая через $A_{n}(u)$ величину $\left.1-2 \sqrt{2 M_{1}} / \sqrt{a u \psi^{-1}\left(\log \left(\log a^{-n}+e\right)\right)}\right)$ :

$$
P_{n}(u) \leqslant 2 \exp \left\{-\psi\left[A_{n}(u) a u \psi^{-1}\left(\log \left(\log a^{-n}+e\right)\right)\right]\right\} .
$$

Далее доказательство разветвляется; докажем сначала (4). Так как $\psi(x)$ - выпуклая функция, то для всех неотрицательных чисел $\alpha$ и $\beta$ таких, что $\alpha+\beta=1$, и для любых $y_{1}$ и $y_{2}$ справедливо неравенство $\psi\left(\alpha y_{1}+\beta y_{2}\right) \leqslant \alpha \psi\left(y_{1}\right)+\beta \psi\left(y_{2}\right)$. В частности, если принять $y_{1}=0$, то, учитывая, что $\psi(0)=0$, имеем: $\psi\left(\beta y_{2}\right) \leqslant \beta \psi\left(y_{2}\right)$; обозначая $\beta y_{2}$ через $x$ и $1 / \beta$ через $\gamma, \gamma \geqslant 1$, получим:

$$
\psi(\gamma x) \geqslant \gamma \psi(x) .
$$

Учитывая (7), из (6) получаем при $A_{n}(u) a u \geqslant 1$ :

$$
P_{n}(u) \leqslant 2 \exp \left\{-A_{n}(u) a u \log \left(\log a^{-n}+e\right)\right\}=2\left(n \log a^{-1}+e\right)^{-A_{n}(u) a u} .
$$

Для доказательства достаточно установить, что для любого $\varepsilon \in\left(0, \frac{1}{4}\right)$ с вероятностью 1 при всех достаточно больших $n$

$$
\sup _{h \in\left[a^{n+1}, a^{n}\right)} \frac{\omega(h)}{g(h)}>1+\varepsilon .
$$

Оценим вероятность события, противоположного (8):

$$
\begin{aligned}
Q_{n}(\varepsilon) & =\mathbf{P}\left\{\sup _{h \in\left[a^{n+1}, a^{n}\right)} \frac{\omega(h)}{h \psi^{-1}(\log (|\log h|+e))}>1+\varepsilon\right\} \\
& \leqslant \mathbf{P}\left\{\frac{\omega\left(a^{n}\right) a^{-n-1}}{\psi^{-1}\left(\log \left(\log a^{-n}+e\right)\right)}>1+\varepsilon\right\} \\
& =\mathbf{P}\left\{\sup _{t \in T(n)}\left(\xi(t)-\xi\left(t_{0}\right)\right) a^{-n}>(1+\varepsilon) a \psi^{-1}\left(\log \left(\log a^{-n}+e\right)\right)\right\} \\
& =P_{n}(1+\varepsilon) \leqslant 2(n|\log a|+e)^{-A_{n}(\varepsilon) a(1+\varepsilon)}
\end{aligned}
$$

Выбирая теперь $a=(1+\varepsilon / 4)^{-1}$ и $n_{0}$ так, чтобы выполнялось неравенство $A_{n}(1+\varepsilon)>(1+\varepsilon / 4)^{-1}, n>n_{0}$, выводим из (9), что

$$
Q_{n}(\varepsilon) \leqslant 2\left(n \log a^{-1}+e\right)^{-(1+\varepsilon)(1+\varepsilon / 4)^{-2}},
$$

откуда заключаем, что ряд $\sum_{n} Q_{n}(\varepsilon)$ сходится. Утверждение (8), а значит, и (4) следует теперь из леммы Бореля-Кантелли. 
Докажем (5). Будем считать $u$ достаточно большим: $u \geqslant u_{0}>0$; заметим, что при любых значениях величин $u$ и $v$, бо́льших 2 , выполняется неравенство $\psi(u v) \geqslant \psi(u)+\psi(v)$. Учитывая также то, что для вогнутой функции $\psi^{-1}(x)$ справедливо неравенство: $\psi^{-1}(y) \geqslant \psi^{-1}(\gamma y) / \gamma$ при любых неотрищательных $y$ и любых $\gamma>1$, из оценки (6) получаем:

$$
\begin{aligned}
P_{n}(u) & \leqslant 2 \exp \left\{-\psi\left(A_{n}(u) a u \frac{\psi^{-1}\left(\gamma \log \left(\log a^{-n}+e\right)\right)}{\gamma}\right)\right\} \\
& \leqslant 2 \exp \left\{-\psi\left(\frac{A_{n}(u) a u}{\gamma}\right)\right\} \exp \{-\gamma \log (n|\log a|+e)\}
\end{aligned}
$$

Поскольку $A_{n}(u) \geqslant A(u)=1-2 \sqrt{2 M_{1}} / \sqrt{a u \psi^{-1}(1)}$ для всех $n \geqslant 0$, то

$$
P_{L}(u) \leqslant \sum_{n=0}^{\infty} P_{n}(u) \leqslant 2\left(\sum_{n=0}^{\infty}(n|\log a|+e)^{-\gamma}\right) \exp \left\{-\psi\left(\frac{A(u) a u}{\gamma}\right)\right\}
$$

Так как

$$
A(u) \geqslant A=1-2 \sqrt{2 M_{1}} / \sqrt{a u_{0} \psi^{-1}(1)}
$$

при $u \geqslant u_{0}$ и ряд $\sum_{n=0}^{\infty}(n|\log a|+e)^{-\gamma}$ сходится, то, обозначая его сумму через $C_{5} / 2$, а величину $A a / \gamma$ через $C_{4}$, получаем (5).

3 а м е ч а н и я и п р и м е ы. Нетрудно убедиться, что для винеровского процесса или поля оценка сверху (4) дает точный результат. Очень простой пример -- процесс $\xi(t)=\xi t$, где $\xi$ - случайная величина из класса $B\left(\lambda^{2} / 2\right)$, для которого $d(t, s)=|t-s|$,

$$
\limsup _{t \rightarrow 0} \frac{\xi(t)}{t \sqrt{\log |\log t|}}=0
$$

- показывает, однако, что верхний предел в (4) может равняться нулю.

Пусть теперь $\xi(t)$ - гауссовское стационарное центрированное поле с ковариационной функцией вида

$$
r(t)=1-\beta|t|^{\alpha}(1+o(1)), \quad t \rightarrow 0, \quad \alpha \in(0,2], \quad \beta>0 .
$$

Здесь $t=\left(t_{1}, t_{2}, \ldots, t_{k}\right),|t|=\left(\sum_{m=1}^{k} t_{m}^{2}\right)^{1 / 2}$. Тогда с вероятностью 1

$$
\limsup _{t \rightarrow t_{0}} \frac{\left|\xi(t)-\xi\left(t_{0}\right)\right|}{\left|t-t_{0}\right|^{\alpha / 2} \sqrt{\log |\log | t-t_{0}||}} \leqslant 2 \sqrt{\beta}
$$

Если же $r(t)$ удовлетворяет условию

$$
r(t)=1-\beta \log ^{-(1+\alpha)}\left(\frac{1}{t}\right)(1+o(1)), \quad \alpha, \beta>0
$$

To

$$
\limsup _{t \rightarrow t_{0}} \frac{\left|\xi(t)-\xi\left(t_{0}\right)\right|}{|\log t|^{-(1+\alpha) / 2} \sqrt{\log \log |\log t|}} \leqslant 2 \sqrt{\beta}
$$


Предположим теперь, что функпия $\varphi(\lambda)$ имеет вид $\varphi(\lambda)=\lambda^{p} / p$, $\lambda>1, p>1$. Если выполнено условие

$$
\int_{0}^{1} \mathcal{H}^{1 / q}(x) d x<\infty, \quad p^{-1}+q^{-1}=1
$$

то, по теореме 1 ,

$$
\limsup _{h \rightarrow 0+} \frac{\omega(h)}{h \sqrt[q]{\log (|\log h|+e)}} \leqslant \sqrt[q]{q} \quad \text { (п.н.) }
$$

и при этом $P_{L}(u) \leqslant C_{5} \exp \left\{-C_{4} u^{q}\right\}, u \geqslant u_{6}$.

В частности, если $T$ - ограниченное выпуклое подмножество $\mathbf{R}^{k}$ и $d(t, s) \leqslant|t-s|^{\alpha}$, то

$$
\limsup _{t \rightarrow t_{0}} \frac{\left|\xi(t)-\xi\left(t_{0}\right)\right|}{\left|t-t_{0}\right|^{\alpha} \sqrt[q]{\log \left(|\log | t-t_{0}||+e\right)}} \leqslant \sqrt[q]{q} \quad \text { (п.н.). }
$$

3. Глобальные верхние функции. Обозначим

$$
M_{2}=\int_{0}^{1} \frac{\mathcal{H}_{2}(x)}{\chi\left(\mathcal{H}_{2}(x)\right)} d x
$$

Сходимость интеграла (10) есть достаточное условие непрерывности $\xi(t)$ в метрике $d$ на метрическом предкомпакте $(T, d)[7]$.

Теорема 2. Пусть поле $\xi(t)$ локально однородно на множестве $T$ и сходится интеграл (10), т.е. $M_{2}<\infty$. Положим

$$
f(h)=h \psi^{-1}(\mathcal{H}(T, h)+\log (|\log h|+e)) .
$$

Тогда с вероятностью 1

$$
\limsup _{h \rightarrow 0+} \frac{\Delta(h)}{f(h)} \leqslant 4
$$

При этом

$$
P_{G}(u)=\mathbf{P}\left\{\sup _{h \in(0 ; 1)} \frac{\Delta(h)}{f(h)}>u\right\} \leqslant C_{8} e^{-\psi\left(C_{7} u\right)} .
$$

Д о к а 3 а т е л ь с т в о. Обозначим через $S(\varepsilon)$ любую из наименьших $\varepsilon$-сетей множества $T$; число ее элементов равно $N(T, \varepsilon)$. Имеем:

$$
\begin{aligned}
\Delta(h) & =\sup _{t \in T} \sup _{s \in B(t, h)}|\xi(t)-\xi(s)|=\sup _{q \in S(h)} \sup _{t \in B(q, h)} \sup _{s \in B(t, h)}|\xi(t)-\xi(s)| \\
& \leqslant \sup _{q \in S(h)}\left\{\sup _{t \in B(q, h)}|\xi(t)-\xi(q)|+\sup _{s \in B(t, h)}|\xi(s)-\xi(q)|\right\} \\
& \leqslant 2 \sup _{q \in S(h)} \sup _{t \in B(q, 2 h)}|\xi(t)-\xi(q)| .
\end{aligned}
$$


Пусть теперь $h \in\left[a^{n+1}, a^{n}\right)$, где $a$, как и ранее, - произвольное число из интервала $(0,1)$; тогда

$$
\Delta(h) \leqslant \Delta\left(a^{n}\right) \leqslant 2 \sup _{q \in S\left(a^{n}\right)} \sup _{t \in B\left(q, 2 a^{n}\right)}|\xi(t)-\xi(q)| .
$$

Введем также обозначения: $\eta_{n}(t, q)=(\xi(t)-\xi(q)) a^{-n} / 2$,

$$
\begin{aligned}
J(n) & =\int_{0}^{1} \frac{\mathcal{H}\left(B\left(q, 2 a^{n}\right), d_{n q}, x\right)}{\chi\left(\mathcal{H}\left(B\left(q, 2 a^{n}\right), d_{n q}, x\right)\right)} d x \\
P_{n}(u, q) & =\mathrm{P}\left\{\sup _{t \in B\left(q, 2 a^{n}\right)}\left|\eta_{n}(t)\right|>u a \psi^{-1}\left(\mathcal{H}\left(T, a^{n}\right)+\log \left(\left|\log a^{n}\right|+e\right)\right)\right\} \\
d_{n q} & =d_{n q}(t, s)=\left\|\eta_{n}(t)-\eta_{n}(s)\right\|, \quad t, s \in B\left(q, 2 a^{n}\right) .
\end{aligned}
$$

В силу локальной однородности поля $\xi(t)$ на множестве $T$ для всех $q \in T$ выполнены соотношения

$$
\begin{aligned}
\mathcal{H}\left(B\left(q, 2 a^{n}\right), d_{n q}, \varepsilon\right) & =\mathcal{H}\left(B\left(q, 2 a^{n}\right), \frac{d a^{-n}}{2}, \varepsilon\right) \\
& =\mathcal{H}\left(B\left(q, 2 a^{n}\right), d, 2 \varepsilon a^{n}\right) \leqslant \mathcal{H}_{2}(\varepsilon)
\end{aligned}
$$

Поэтому

$$
J(n) \leqslant \int_{0}^{1} \frac{\mathcal{H}_{2}(x)}{\chi\left(\mathcal{H}_{2}(x)\right)} d x=M_{2}<\infty .
$$

Так как $\left\|\eta_{n}(t, q)\right\| \leqslant 1, t \in B\left(q, 2 a^{n}\right)$, то цля оценки вероятности $P_{n}(u, q)$ применимо неравенство Дмитровского [5], когда

$$
u a \psi^{-1}\left(\mathcal{H}\left(T, a^{n}\right)+\log \left(\left|\log a^{n}\right|+e\right)\right) \geqslant C_{6}
$$

Производя аналогичные сделанным при доказательстве теоремы 1 рассуждения и обозначая через $A_{n}(u)$ величину

$$
1-\frac{2 \sqrt{2 M_{2}}}{a u \psi^{-1}\left(\mathcal{H}\left(T, a^{n}\right)+\log \left(\left|\log a^{n}\right|+e\right)\right)},
$$

получаем неравенство

$$
P_{n}(u, q) \leqslant 2 \exp \left\{-\psi\left(A_{n}(u) a u \psi^{-1}\left(\mathcal{H}\left(T, a^{n}\right)+\log \left(\left|\log a^{n}\right|+e\right)\right)\right)\right\} .
$$

Докажем (11). Пусть $A_{n}(u) a u \geqslant 1$, тогда, учитывая (7), из (13) получаем

$$
\begin{aligned}
P_{n}(u, q) & \leqslant 2 \exp \left\{-A_{n}(u) a u\left(\mathcal{H}\left(T, a^{n}\right)+\log \left(\left|\log a^{n}\right|+e\right)\right)\right\} \\
& =2\left(N\left(T, a^{n}\right)(n|\log a|+e)\right)^{-A_{n}(u) a u .} .
\end{aligned}
$$


Для доказательства (11) достаточно показать, что при всех достаточно больших $n$ для любого $\varepsilon \in\left(0, \frac{1}{4}\right)$

$$
\sup _{h \in\left[a^{n+1}, a^{n}\right)} \frac{\Delta(h)}{f(h)} \leqslant 4(1+\varepsilon) .
$$

Оценим вероятность противоположного события:

$$
\begin{aligned}
& Q_{n}(\varepsilon)=\mathbf{P}\left\{\sup _{h \in\left[a^{n+1}, a^{n}\right)} \frac{\Delta(h)}{h \psi^{-1}(\mathcal{H}(T, h)+\log (|\log h|+e))}>4(1+\varepsilon)\right\} \\
& \leqslant \mathbf{P}\left\{\frac{\Delta\left(a^{n}\right) a^{-n-1}}{\psi^{-1}\left(\mathcal{H}\left(T, a^{n}\right)+\log \left(\left|\log a^{n}\right|+e\right)\right)}>4(1+\varepsilon)\right\} \\
& \leqslant \mathbf{P}\left\{\bigcup_{q \in S\left(a^{n}\right)} \sup _{t \in B\left(q, 2 a^{n}\right)} \frac{2|\xi(t)-\xi(q)| a^{-n-1}}{\psi^{-1}\left(\mathcal{H}\left(T, a^{n}\right)+\log \left(\left|\log a^{n}\right|+e\right)\right)}>4(1+\varepsilon)\right\} \\
& \leqslant N\left(T, a^{n}\right) \mathbf{P}\left\{\sup _{t \in B\left(q, 2 a^{n}\right)}\left|\eta_{n}(t, q)\right|>a(1+\varepsilon)\right. \\
& \left.\quad \times \psi^{-1}\left(\mathcal{H}\left(T, a^{n}\right)+\log \left(\left|\log a^{n}\right|+e\right)\right)\right\} \\
& =N\left(T, a^{n}\right) P_{n}(1+\varepsilon, q) \\
& \leqslant 2 N\left(T, a^{n}\right)^{1-A_{n}(1+\varepsilon) a(1+\varepsilon)}(n|\log a|+e)^{-A_{n}(1+\varepsilon) a(1+\varepsilon)} .
\end{aligned}
$$

Выбирая теперь $a$ и $n_{0}$ так же, как и при доказательстве теоремы 1 , замечаем, что $\sum_{n} Q_{n}(\varepsilon)<\infty$ при всех $\varepsilon \in\left(0, \frac{1}{4}\right)$, откуда, на основании леммы Бореля-Кантелли, следует (11). Докажем теперь (12). Пусть $u$ достаточно большое: $u \geqslant u_{0}>0$. Тогда из (13) выводим:

$$
\begin{aligned}
P_{n}(u, q) & \leqslant 2 \exp \left\{-\psi\left(A_{n}(u) a u \frac{\psi^{-1}\left(\gamma \mathcal{H}\left(T, a^{n}\right)+\gamma \log \left(\left|\log a^{n}\right|+e\right)\right)}{\gamma}\right)\right\} \\
& \leqslant 2 \exp \left\{-\psi\left(\frac{A_{n}(u) a u}{\gamma}\right)\right\} N\left(T, a^{n}\right)^{-\gamma}(n|\log a|+e)^{-\gamma}
\end{aligned}
$$

для всех $\gamma>1$. Поскольку. при $n \geqslant 0, u \geqslant u_{0}$

$$
A_{n}(u) \geqslant A=1-\frac{2 \sqrt{2 M_{2}}}{\sqrt{a u_{0} \psi^{-1}(1)}}
$$

то

$$
\begin{aligned}
P_{G}(u) \leqslant & 2 \sum_{n=0}^{\infty} N\left(T, a^{n}\right) P_{n}(u, q) \leqslant 2\left(\sum_{n=0}^{\infty}(n|\log a|+e)^{-\gamma}\right) \\
& \times \exp \left\{-\psi\left(\frac{A a u}{\gamma}\right)\right\} .
\end{aligned}
$$

Так как для любых $\gamma>1$ ряд $\sum_{n=0}^{\infty}(n|\log a|+e)^{-\gamma}$ сходится, то, обозначая его сумму через $C_{8} / 2$, а величину $A a / \gamma$ через $C_{7}$, получаем (12). 
Рассмотрим пример. Пусть, как и в п. $2, \xi(t)$ - гауссовские стационарные поля, $T=\left\{t: t \in \mathbf{R}^{k},|t| \leqslant 1\right\}$,

$$
r(t)=1-\beta|t|^{\alpha}(1+o(1)), \quad t \rightarrow 0
$$

По теореме 2 с учетом того обстоятельства, что $\mathcal{H}(h) \sim k \alpha^{-1}|\log h|$, $h \rightarrow 0$, находим:

$$
f(h)=\left(2 k \alpha^{-1}\right)^{1 / 2} h|\log h|^{1 / 2}, \quad \limsup _{h \rightarrow 0+} \frac{\Delta(h)}{f(h)} \leqslant 4 .
$$

Аналогично анализируются остальные примеры п. 2. Заметим, впрочем, что оценки типа (12) в гауссовском случае вытекают и из теоремы Ферника [8].

4. Некоторые приложения. 1) ДПТ в банаховом пространстве. Пусть $T$ - произвольное множество, $\xi_{i}(t)$ - независимые центрированные одинаково распределенные случайные поля,

$$
\varphi(\lambda) \leqslant \frac{\lambda^{2}}{2}, \quad S_{n}(t)=n^{-1 / 2} \sum_{i=1}^{n} \xi_{i}(t), \quad d(t, s)=\left\|\xi_{n}(t)-\xi_{n}(s)\right\|
$$

Если $\mathcal{H}_{2}(\varepsilon) \leqslant \mathcal{H}_{0}+\boldsymbol{x}|\log \varepsilon|, \varepsilon<1$, то поля $S_{n}(t)$ слабо сходятся в топологии Прохорова-Скорохода в пространстве $C(T, d)[9]$.

Поскольку $\left\|S_{n}(t)-S_{n}(s)\right\| \leqslant d(t, s)$, то по теореме 2 функция $f(h)=$ $h|\log h|^{1 / 2}$ будет глобальной верхней функцией для всех $S_{n}(t)$, и равномерно по $n$ будет выполнено неравенство $(12)$ с заменой $\xi(t)$ на $S_{n}(t)$. Следовательно, $\xi_{i}(t)$ удовлетворяют ЦПТ также в пространстве Гёльдера с нормой

$$
\|x(t)\|=\sup _{t \in T}|x(t)|+\sup _{h \in(0,1)} \frac{\Delta_{x}(h)}{h^{1-\beta}}, \quad \beta \in(0,1) .
$$

2) Метод Монте-Карло. Пусть на некотором измеримом пространстве $(X, \mathcal{F})$ с вероятностной мерой $\mu_{\xi}$ определен интеграл

$$
J(t)=\int_{X} f(t, x) \mu_{\xi}(d x)
$$

В методе Монте-Карло МЗИ-приближением [10], [11] является случайное поле

$$
J_{n}(t)=n^{-1} \sum_{i=1}^{n} f\left(t, \xi_{i}\right)
$$

где $\xi_{i}$ - независимые величины в $X$ с распределениями $\mu_{\xi}$. На практике по формуле $(15)$ значения $J_{n}(t)$ вычисляются в некоторой конечной сетке значений $t$, и знание ощенок для модуля непрерывности, например, 
согласно (12), позволит оценить возникаюшую при этом погрешность интерполяции .

\section{СПИСОК ЛИТЕРАТУРЫ}

1. Леви П. Стохастические процессы и броуновское движение. М.: Наука, 1972, $375 \mathrm{c}$.

2. Петров $B$. В. Суммы независимых случайных величин. М.: Наука, 1972, 414 с.

3. Ядренко М. И. Локальні властивості вибіркових функцій випадкових полів. Вісн. Київського університету, сер. мат. та мех., 1967, № 9, с. 103-112.

4. Альбин Дж. М. П. Верхние и нижние классы для гауссовских случайных полей и обобщенный захон повторного логарифма. - Теория вероятн. и ее примен., 1992, т. 37, в. 1, с. 5-9.

5. Дмитровский $B$. А. О распределении максимума и локальных свойствах реализаций предгауссовских полей. - Теория вероятн. и мат. статист., 1981, в. 25, c. $154-164$.

6. Fernique $X$. Sur la régularité de certaines classes de fonctions aléatoires. - C. R. Acad. Sci. Paris, Série 1, 1988, t. 307, p. 493-496.

7. Козаченко Ю. В., Островский Е. И. Банаховы пространства случайных величин типа субгауссовских. - Теория вероятн. и матем. статист., 1985, в. 32 , c. $42-53$.

8. Fernique $X$. Régularité des trajectoires des fonctions aléatoires gaussiennes. - Lect. Notes Math., 1975, v. 480, p. 1-245.

9. Дмитровский В. А., Ермаков С. В., Островский Е. И. Центральная предельная теорема для слабозависимых величин со значениями в банаховом пространстве. - Теория вероятн. и ее примен., 1983, т. XXVIII, в. 1, с. 83-96.

10. Фролов $A . C$., Ченчов $H$. Н. О вычислении методом Монте-Карло определенных интегралов, зависящих от параметра. -- Журн. вычисл. матем. и матем. физики, 1962 , т. 2, № 4, с. 714-717.

11. Дмитровский $B$. A., Островский $Е$. И. Оценка погрешности метода зависимых испытаний. - Журн. вычисл. матем. и матем. физики, 1976, т. 18, № 5, с. 13121316. 